\title{
Tiny cystine stones in the gallbladder of a patient with cholecystolithiasis complicating acute cholecystitis: a case report
}

\author{
Tie Qiao ${ }^{1,2,3^{*}}$, Rui-hong Ma ${ }^{1,2}$, Xiao-bing Luo ${ }^{1,2}$, Yu-yang Feng ${ }^{1,2}$, Xing-qiang Wang ${ }^{1,2}$, Pei-ming Zheng ${ }^{1,2}$ and \\ Zhen-liang Luo ${ }^{1,2}$
}

\begin{abstract}
Cystine stones, the main component of which is cystine, are very common urinary calculi, but are rare in the gall bladder. In animals, there has been only one report of cystine gallstones in tree shrews, and to our knowledge, this is the first report of cystine gallstones in humans.
\end{abstract}

Keywords: Cystine stones, Urinary calculi, Gallstones

\section{Background}

Cholecystolithiasis, or gallbladder stone is a common and frequently encountered disease worldwide [1-4]. Gallbladder stones can be divided into four groups according to their location: intracavitary, cystic duct, intramural, and mucosal stones (small stones adhering to the mucosa) [5]. Based on their main component, gallbladder stones are divided into cholesterol stones, bile pigment stones, mixed stones, and other types [6-9] (including calcium carbonate stones, calcium phosphate stones, fatty acid stones, and cystine stones); this classification is mainly based on the infrared spectrum of the stones. Compared with other types of stones, cystine stones are rare in the gallbladder, although they are common in the urinary system. We report a patient with cystine gallstones.

\section{Case presentation}

A 38-year-old woman with a 3-year history of cholecystolithiasis was examined at a local hospital and referred to our hospital for endoscopic gallstone removal without gallbladder excision. When she was hospitalized at our hospital, the acute right upper abdominal pain had persisted for two hours.

\footnotetext{
* Correspondence: faj1958@163.com

'Institute of Gallbladder Disease of Panyu, Guangzhou 511470, People's Republic of China

Full list of author information is available at the end of the article
}

On physical examination, no signs of jaundice were seen in the skin or sclera. The patient's abdomen was soft, with no sign of lumps, with tenderness other than rebound tenderness in the gallbladder area. Murphy's sign was positive. Ultrasonography revealed several movable masses of $7 \times 10 \mathrm{~mm}$ and $7 \times 9 \mathrm{~mm}$ in size, which were strongly echogenic, with acoustic shadowing in the body of the gallbladder; an immovable strongly echogenic mass of $7 \times 10 \mathrm{~mm}$ with acoustic shadowing in the neck of gallbladder and cystic duct; and poor sound penetration and a dense, low-light spot in the dark space of the bile. There was no thickening of the gallbladder wall. The gallbladder emptying index was $30 \%$. No obvious abnormalities were seen in the liver, spleen or pancreas.

The patient was diagnosed as having cholecystolithiasis complicating acute cholecystitis and incomplete biliary-tract obstruction. The patient had been given antiinflammatory treatment after the examination for 2 weeks and the symptoms of acute cholecystitis had disappeared before the operation was schedule, and the patient was strongly in favor of preserving the gallbladder.

Consequently, after approval by the medical ethics committee and provision of informed consent by the patient, the gallbladder was laparoscopically isolated and transected at the bottom $(<6 \mathrm{~mm})$ under general anesthesia. First, the bile was drained with a sterile ventricular drainage tube to a sterile injector, and 
transferred to sterile tubes. Next, the gallbladder was explored with a three-channeled cholecystoscope (CHiAO; Chinese national patent number: ZL200810026985.X HAWK, China [10]), and the stones were collected with a stone extractor. We found only mild congestion in the gallbladder mucosa, which indicated only slight inflammation of gallbladder. Using endoscopy, we found many small, semitransparent stones $(<1 \mathrm{~mm})$ adhered to the gallbladder wall (Figure $1 \mathrm{~A}, \mathrm{~B})$. These stones were removed with an endoscopic attachment (CHiAO absorbing box; Chinese national patent number: ZL 201110167069.X) designed to remove sludge-like gallstones combined with seven types of manipulation (pushing, squeezing, pressuring, tearing, bracing, flushing, and sucking) [5], while several large stones $(>5 \mathrm{~mm})$ were removed with a stone extractor (Figure 1C, D). The small stones were yellowish and semitransparent, and the large stones were polyhedron or globular in shape, and had a radial, layered arrangement in profile (Figure $1 \mathrm{E}-\mathrm{H}$ ). The bile was yellowish, opaque, turbid, and very viscous. After centrifugation at $1,450 \mathrm{~g}$ for $10 \mathrm{~min}$, the bile supernatant was transferred to a clean tube, and about $0.5 \mathrm{~mL}$ of sediment was kept. The bile sediment was smeared onto labeled slides and viewed under a system microscope (BX51; Olympus, Tokyo, Japan). Colorless hexagonal plate crystals with high refractivity and limpid edges were seen, which were judged to be cystine crystals based on their morphology. Meanwhile, colorless, transparent crystals (in the shape of rectangles with missing corners or squares with missing corners, or glass flakes) were seen, which were judged to be cholesterol crystals from their morphology (Figure 2).

The gallbladder stones were analyzed with a Fourier transform infrared spectrometer (TENSOR27; Bruker Optics GmBH, Ettlingen, Germany) in the frequency range of 400 to 4,000 per $\mathrm{cm}$ at 4 per $\mathrm{cm}$ resolution. The results indicated that the small stones were cystine stones and that the large stones were cholesterol stones; (Figure 3A, B).

Next, some of the small stones and a piece of a large stone were fixed onto the sample table and dried at $60^{\circ}$ C overnight, then sputter-coated with gold (ETD-2000, Beijing Elaborate Technology Development Ltd., China) and observed under a scanning electron microscope (EVO LS10; Carl Zeiss, Cambridge, England). The samples were photographed and analyzed with an energy spectrometer (X-Max; Oxford Instruments plc, Oxford, UK). Under scanning electron microscopy, the small stones were found to be composed of hexagonal cystine crystals $(30-270 \mu \mathrm{m})$, some with prominences on their edges. The energy spectrum indicated that the elemental composition was carbon, oxygen, nitrogen, and sulfur, along with gold from the coating. The large stones were composed of lamellar cholesterol crystals with a thickness of about $1 \mu \mathrm{m}$. The energy spectrum indicated that the elemental composition was carbon and oxygen, with gold from the coating (Figure $3 \mathrm{C}-\mathrm{S}$ ).

\section{Conclusions}

The spectrum of the small stones indicated that the main component was cystine, and the energy spectrum revealed that the main elements were carbon, oxygen, nitrogen, and sulfur, confirming that these stones were composed of cystine. The spectrum of the large stones indicated that the main component was cholesterol, and the energy spectrum confirmed this, showing that the main elements were carbon and oxygen.

There are no reports of cystine gallstones in any species, with the exception of one report describing sick tree shrews [11], and in that case, the pathogenesis was not clear. There have been no reports in humans. Urinary cystine stones are mainly induced by cystinuria, an inborn error of metabolism [12-15]. Supersaturation of cystine in the urine leads to the precipitation of cystine crystals, and subsequent formation of cystine stones. We found cystine crystals in our patient's bile sediment, which indicated that the pathogenesis of cystine stones in the gallbladder may be similar to that in the urinary system. However, the patient did not have urinary calculi. It is possible that the presence of stones in the cystic duct led to the incomplete obstruction of the biliary tract and a change in bile metabolism, resulting in the formation of cystine stones.

The mechanism of the formation of cholesterol gallstones involves three main components: supersaturation of cholesterol, gallbladder hypomotility, and kinetic factors [16-26]. Cholesterol is slightly soluble in aqueous media, but is made soluble in bile by forming mixed micelles with bile salts and lecithin. An increase in lecithin or a decrease in bile acid will lead to supersaturation of cholesterol, which produces crystals and precipitates around the core, with spiral growth from the center outwards, and a radial, layered, cord-like arrangement of cholesterol crystals, culminating in stone formation. Patients with incomplete gallbladder emptying were found to have increased total lipid concentrations and some proteins that promote stone formation [20]. The main mechanism for the formation of cholesterol stones, which involves cholesterol supersaturation, gallbladder hypomotility, and kinetic factors, may also lead to the formation of other types of gallstones. Our patient had a normal blood lipid level and a gallbladder emptying index of $30 \%$, indicating poor gallbladder function. These factors may be involved in the formation of both cholesterol and cystine stones. 

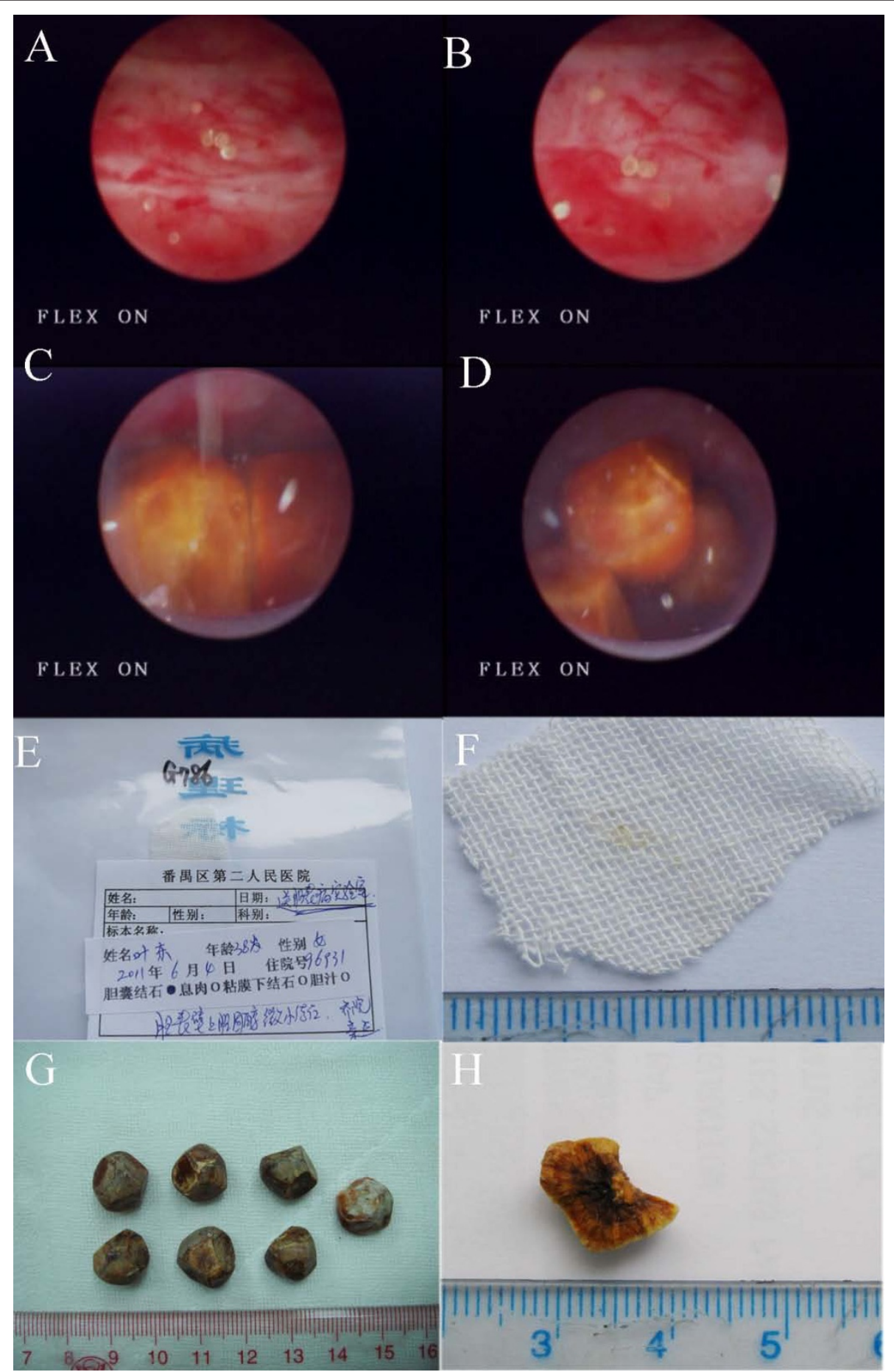

Figure 1 Endoscopic and gross appearance of the stones. (A,B) Small stones and purulent bile and (C,D) large stones and purulent bile under endoscopy. $(\mathbf{E}-\mathbf{H})$ Appearance of $(\mathbf{E}, \mathbf{F})$ small stones and $(\mathbf{G}, \mathbf{H})$ large stones with a radial, layered arrangement in profile.

In conclusion, we report a case of cystine gallstones, a rare type of gallstone, combined with cholesterol stones, in a patient with cholecystolithiasis complicating acute cholecystitis and incomplete obstruction of the biliary duct. The tiny cystine gallstones were adhered to the gallbladder wall and therefore difficult to locate. Surgeons should be aware of the possibility of such stones and ensure that they are removed to reduce the possibility of recurrence. The patient was followed up for 6 months with no abnormal occurrence or recurrence, 


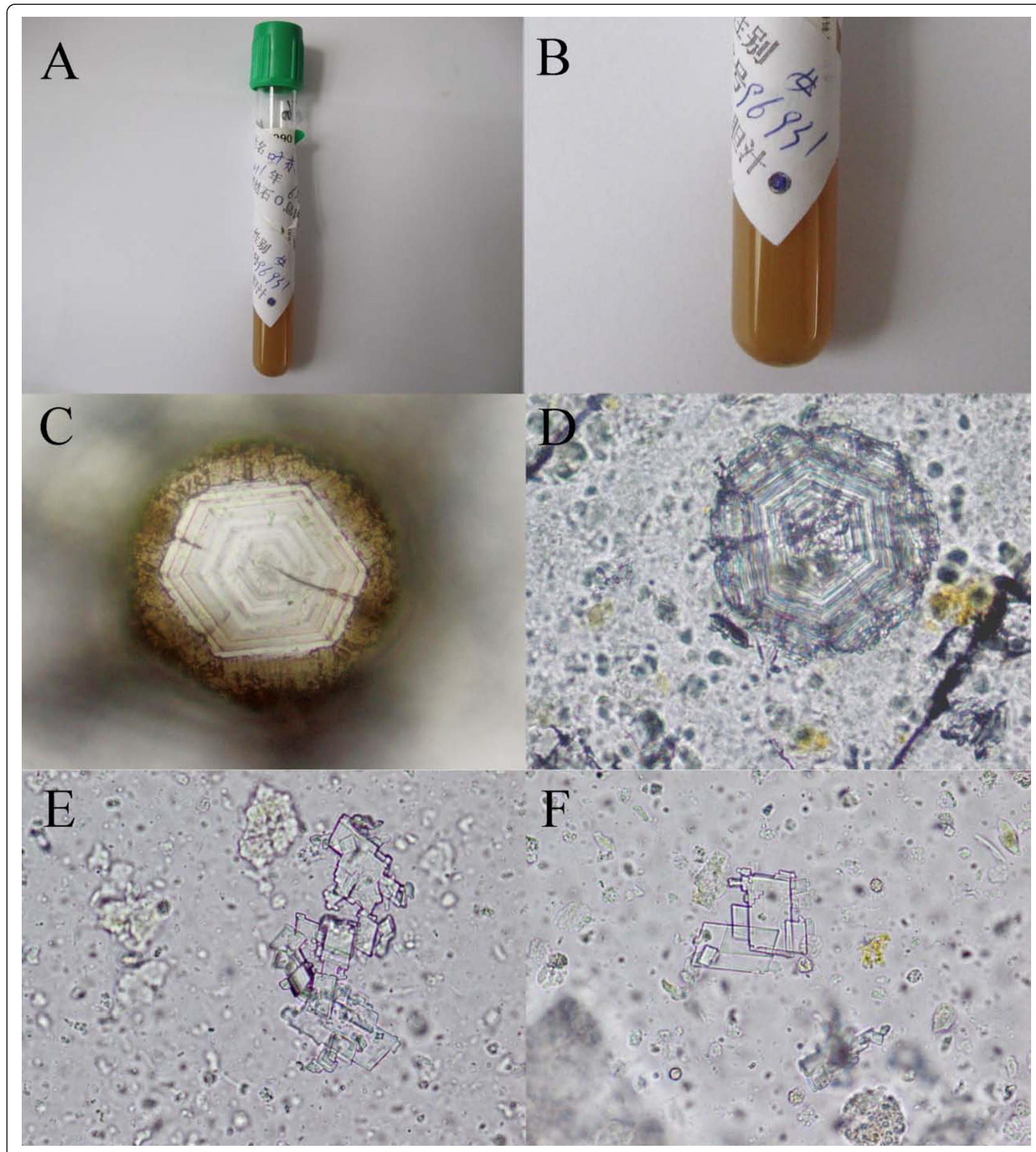

Figure 2 Appearance and microscopic examination of the bile. $(\mathbf{A}, \mathbf{B})$ Appearance of the bile. $(\mathbf{C}, \mathbf{D})$ Cystine and $(\mathbf{E}, \mathbf{F})$ cholesterol crystals under light microscopy.

indicating that the gallstone removal without gallbladder excision has been effective, but she will need to have a longer period of follow-up.

The pathogenesis of cystine stone formation in the gallbladder is not clear, and requires further research.
However, this case report provides new insight into the pathogenesis of gallstones. The present research indicates that the composition and genesis of gallstones is more complicated than previously thought, and requires further research and exploration. 

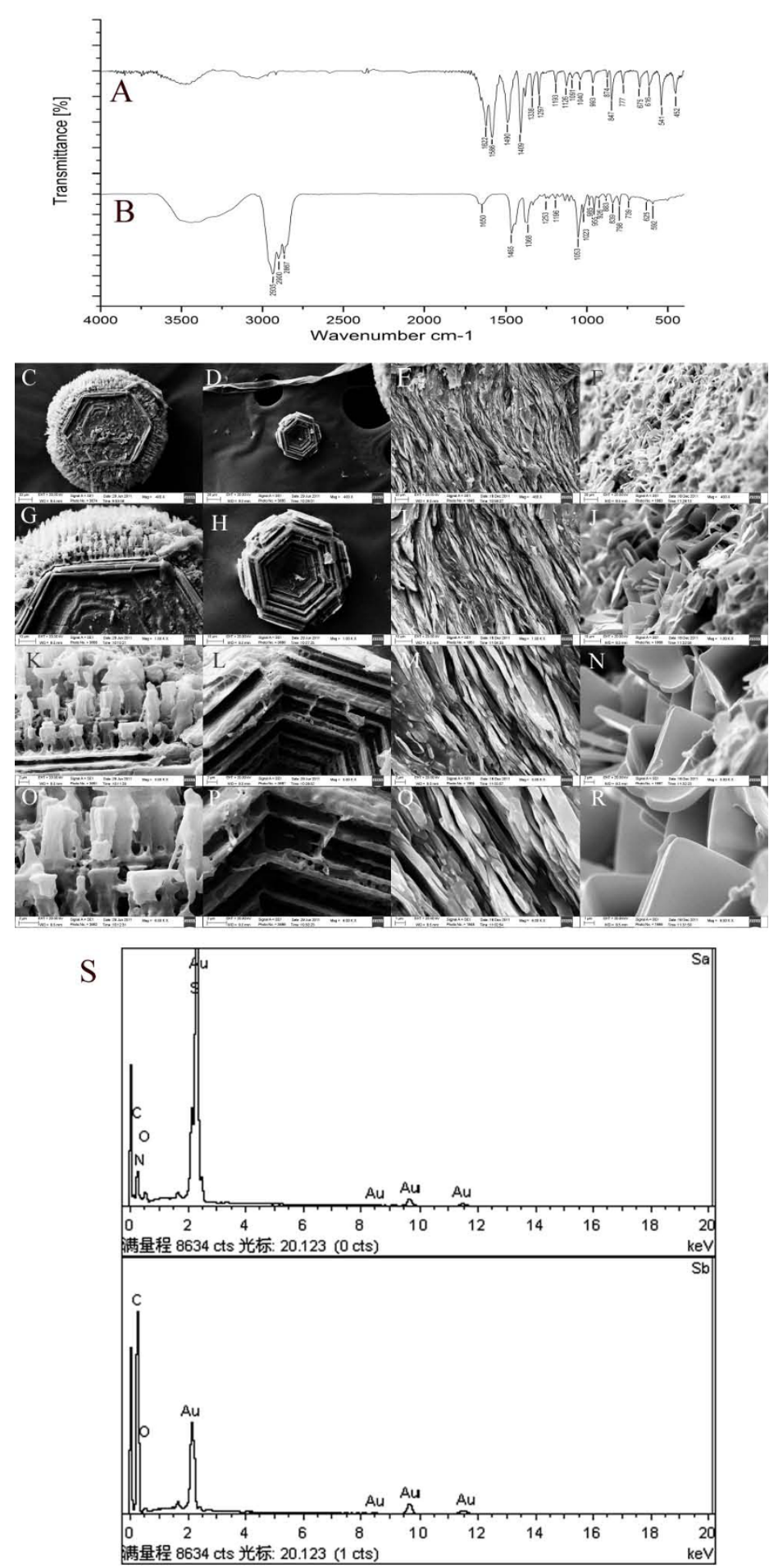

Figure 3 Results of analysis with spectrogram and electron microscope. (A,B) FTIR spectrogram of (A) small stones, showing that the main component was cystine, and (B) of the large stones, showing that the main component was cholesterol. (C-R) Scanning electron microscopy of the small cystine and large cholesterol stones. The small cystine stones displayed (C,D) hexagon crystals of unequal size, some with prominences on the edge (original magnification $\times 400)$ and $(\mathbf{G}, \mathbf{H}, \mathbf{K}, \mathbf{L})$ hexagon crystals stacked and arranged tightly. Original magnification $(G, H) \times 1,000 ;(K, L)$ $\times 3,000$. $(\mathbf{O}, \mathbf{P}) \mathrm{A}$ view of one corner of a hexagon crystal shows that the end of the prominence is lamellar (original magnification $\times 6,000)$. The large cholesterol stones showed $(\mathbf{E}, \mathbf{F})$ amellar cholesterol crystals (original magnification $\times 400$ ) and $(\mathbf{I}, \mathbf{J}, \mathbf{M}, \mathbf{N})$ stacked lamellar cholesterol crystals, Original magnification $(I, J) \times 1,000 ;(\mathrm{M}, \mathrm{N}) \times 3,000$. $(\mathbf{Q}, \mathbf{R})$ The cholesterol crystals had a smooth, glossy surface, dull borders, and a pyknomorphous texture (original magnification $\times 6,000$ ). (S) X-ray energy spectrogram Sa, spectrogram of the small stones (the elemental composition was carbon, oxygen, nitrogen, and sulfur) Sb, spectrogram of the large stones (the elemental composition was carbon and oxygen). 


\section{Consent}

Written informed consent was obtained from the patient for publication of this Case report and any accompanying images. A copy of the written consent is available for review by the Editor-in-Chief of this journal.

\section{Abbreviations}

CS: Cystine stones; UC: Urinary calculi; GS: Gallstones; CS: Cholesterol stones; BPG: Bile pigment stones; MS: Mixed stones; CCS: Calcium carbonate stones; CPS: Calcium phosphate stones; FAS: Fatty acid stones; FTIR: Fourier transform infrared spectroscopy; SEM: Scanning electron microscope; XRES: $\mathrm{X}$-ray energy spectrometer.

\section{Acknowledgements}

We are grateful to Xiao-feng Wang and Gang Wang in the General Surgery Department of our hospital for their kindly support in providing specimens. We thank other colleagues in the General Surgery Department and the operating room for their enthusiastic help, and the leaders of our hospital for their kind support. We thank the Edanz group China for providing editing assistance for our manuscript.

\section{Author details}

${ }^{1}$ Institute of Gallbladder Disease of Panyu, Guangzhou 511470, People's Republic of China. ${ }^{2}$ The Second People's Hospital of Panyu, Panyu, Guangzhou 511470, People's Republic of China. ${ }^{3}$ The Second People's Hospital of Panyu, Xingye Road No. 7, Dagang Town, Panyu, Guangzhou 511470, People's Republic of China.

\section{Authors' contributions}

$\mathrm{TQ}$, study concept and design; RM, acquisition of data; analysis and interpretation of data; drafting of the manuscript; $\mathrm{XL}$, critical revision of the manuscript for important intellectual content; YF, material support; XW, material support; PZ, technical, or material support; ZL, technical, or material support. All authors read and approved the final manuscript.

\section{Authors' information}

Tie Qiao, bachelor's degree, Superintendent of the institution, surgeon; Ruihong Ma, master's degree, researcher; Xiao-bing Luo, master's degree, researcher; Yu-yang Feng, bachelor's degree, surgeon; Xing-qiang Wang, bachelor's degree, surgeon; Pei-ming Zheng, master's degree, researcher; Zhen-liang Luo, doctor's degree, researcher.

\section{Competing interests}

The authors declare that they have no competing interests.

Received: 31 January 2012 Accepted: 29 March 2012

Published: 29 March 2012

\section{References}

1. Kratzer W, Mason RA, Kachele V: Prevalence of gallstones in sonographic surveys worldwide. J Clin Ultrasound 1999, 27:1-7.

2. Everhart JE, Khare M, Hill M, Maurer KR: Prevalence and ethnic differences in gallbladder disease in the United States. Gastroenterology 1999, 117:632-639.

3. Sampliner RE, Bennett PH, Comess L, Rose FA, Burch TA: Gallbladder disease in pima Indians. Demonstration of high prevalence and early onset by cholecystography. N Engl J Med 1970, 283:1358-1364.

4. Everhart JE, Yeh F, Lee ET, Hill MC, Fabsitz R, Howard BV, Welty TK: Prevalence of gallbladder disease in American Indian populations: findings from the strong heart study. Hepatology 2002, 35:1507-1512.

5. Qiao T, Chen XT, Zhang BS, Lu JG: Map of the CHiAO Brand rigid cholecystoscope application (M). The first edition 2010, 19:41.

6. Sossé Djessou P, Aké Mondé A, Tiahou G, Koffi G, Cissé Camara M, Djohan F, Yapo E, Kassayou S, Peuchant E, Essiagne Sess D, Monnet D: Gallstone biochemical characteristics using Fourier transform infrared spectroscopy method. Ann Biol Clin (Paris) 2010, 68(1):39-42.

7. Yoo EH, Oh HJ, Lee SY: Gallstone analysis using Fourier transform infrared spectroscopy (FT-IR). Clin Chem Lab Med 2008, 46(3):376-381.
8. Stringer MD, Soloway RD, Taylor DR, Riyad K, Toogood G: Calcium carbonate gallstones in children. J Pediatr Surg 2007, 42(10):1677-1682.

9. Kleiner O, Ramesh J, Huleihel M, Cohen B, Kantarovich K, Levi C, Polyak B, Marks RS, Mordehai J, Cohen Z, Mordechai S: A comparative study of gallstones from children and adults using FTIR spectroscopy and fluorescence microscopy. BMC Gastroenterol 2002, 2:3.

10. Qiao T, Huang WC, Luo XB, Zhang YD: Design and application of a new series of gallbladder endoscopes that facilitate gallstone removal without gallbladder excision. Rev Sci Instrum 2012, 83(1):015115.

11. Schwaier A: Tupaias (tree shrews)-a new animal model for gallstone research; first observations of gallstones. Res Exp Med (Berl) 1979, 176(1):15-24

12. Ahmed K, Dasgupta $P$, Khan MS: Cystine calculi: challenging group of stones. Postgrad Med J 2006, 82(974):799-801.

13. Evan AP, Coe FL, Lingeman JE, Shao Y, Matlaga BR, Kim SC, Bledsoe SB, Sommer AJ, Grynpas M, Phillips CL, Worcester EM: Renal crystal deposits and histopathology in patients with cystine stones. Kidney Int 2006, 69(12):2227-2235.

14. Purohit RS, Stoller ML: Stone clustering of patients with cystine urinary stone formation. Urology 2004, 63(4):630-635.

15. Tekin A, Tekgul S, Atsu N, Sahin A, Bakkaloglu M: Cystine calculi in children: the results of a metabolic evaluation and response to medical therapy. J Urol 2001, 165(6 Pt 2):2328-2330.

16. Ahlberg J, Curstedt T, Einarsson K, Sjovall J: Molecular species of biliary phosphatidylcholines in gallstone patients: the influence of treatment with cholic acid and chenodeoxycholic acid. J Lipid Res 1981, 22:404-409

17. Admirand WH, Small DM: The physicochemical basis of cholesterol gallstone formation in man. J Clin Invest 1968, 47:1043-1052.

18. Carey MC: Critical tables for calculating the cholesterol saturation of native bile. J Lipid Res 1978, 19:945-955

19. Wang DQ, Carey MC: Characterization of crystallization pathways during cholesterol precipitation from human gallbladder biles: identical pathways to corresponding model biles with three predominating sequences. J Lipid Res 1996, 37:2539-2549.

20. Stolk MF, Van Erpecum KJ, Peeters TL, Samsom M, Smout AJ, Akkermans LM, Vanberge-Henegouwen GP: Interdigestive gallbladder emptying, antroduodenal motility, and motilin release patterns are altered in cholesterol gallstone patients. Dig Dis Sci 2001, 46:1328-1334.

21. Stolk MF, van Erpecum KJ, Renooij W, Portincasa P, van de Heijning BJ, vanBerge-Henegouwen GP: Gallbladder emptying in vivo, bile composition, and nucleation of cholesterol crystals in patients with cholesterol gallstones. Gastroenterology 1995, 108:1882-1888.

22. van Erpecum KJ, Venneman NG, Portincasa P, Vanberge-Henegouwen GP: Review article: agents affecting gall-bladder motility-role in treatment and prevention of gallstones. Aliment Pharmacol Ther 2000, 14(Suppl 2):66-70.

23. Colecchia A, Sandri L, Bacchi-Reggiani ML, Portincasa P, Palasciano G, Mazzella G, Roda E, Festi D: Is it possible to predict the clinical course of gallstone disease? Usefulness of gallbladder motility evaluation in a clinical setting. Am J Gastroenterol 2006, 101:2576-2581.

24. Lee SP, LaMont JT, Carey MC: Role of gallbladder mucus hypersecretion in the evolution of cholesterol gallstones. J Clin Invest 1981, 67:1712-1723.

25. Lee SP, Nicholls JF: Nature and composition of biliary sludge. Gastroenterology 1986, 90:677-686.

26. Ko CW, Schulte SJ, Lee SP: Biliary sludge is formed by modification of hepatic bile by the gallbladder mucosa. Clin Gastroenterol Hepatol 2005, 3:672-678

doi:10.1186/2047-783X-17-6

Cite this article as: Qiao et al:: Tiny cystine stones in the gallbladder of a patient with cholecystolithiasis complicating acute cholecystitis: a case report. European Journal of Medical Research 2012 17:6. 